\title{
2 Medizin und Bewusstsein. Auf dem Weg zu einer Kulturheilkunde?
}

\author{
Hartmut Schröder
}

\section{Was hat Kultur mit Medizin zu tun?}

„Die Kultur ist der Weg der Seele zu sich selbst.“ (Georg Simmel)

Existenz und Berechtigung einer besonderen Naturheilkunde - nicht neben sondern als Teil der Medizin - sind von Politik und Gesellschaft auf vielfältige Weise anerkannt und finden Ausdruck in der Zusatzbezeichnung Naturheilverfahren, die von den Ärztekammern verliehen wird. Der Begriff Kulturheilkunde hingegen ist weitestgehend unbekannt, obwohl er durchaus eine Vorgeschichte ${ }^{3}$ hat und eigentlich Konsens darüber besteht, dass Kultur - was immer das auch sein mag - Einfluss auf Krankheit und Gesundheit sowie Heilung hat. In den letzten Jahren sind zunehmend praktische Initiativen „Kultur heilt“ entstanden, die einen Höhepunkt in der Kampagne „Kultur auf Rezept" der Ärztekammer der finnischen Stadt Turku erhielt. ${ }^{4}$ Gleichzeitig hat die Zahl von Studien zugenommen, die salutogene Effekte kultureller Teilhabe bestätigen. Der Forschungsstand zur Wirksamkeit von Entspannungsverfahren und Mentaltechniken sowie von Achtsamkeit und Meditation ist ebenfalls beeindruckend, wobei diese Methoden auch unter dem Begriff Kulturtechniken zusammengefasst werden können. Es ist also durchaus an der Zeit, zu überlegen, ob und wie das Konzept Kulturheilkunde praktisch und theoretisch weiterentwickelt werden kann.

Für die Spezies Mensch ist das gleichzeitige Dasein als Natur- und Kulturwesen charakteristisch. Herder $(1784: 146)$ hat Menschen als erste „Freigelassene der

3 Siehe dazu den Überblick in Schröder/Mildenberger (2013).

Siehe dazu die Übersicht in Pick (2011). 
Schöpfung“ bezeichnet. Sie sind durch ihr Bewusstsein und ihren Geist dazu in der Lage, ihre Umwelt zu gestalten und Kultur zu schaffen, womit sie über ihre eigene Natur hinausgehen und sich selber kultivieren. Dies eröffnet Menschen neue Wirkmöglichkeiten und gibt ihnen eine gewisse Vormachtstellung im Reich der Lebewesen, was allerdings Fluch und Segen zugleich sein kann. Menschen können sich in einem bestimmten Rahmen dank ihrer kulturellen Entwicklung zwar selber heilen, aber sie können sich über ihr Bewusstsein auch selber krank machen.

\footnotetext{
Wenn man als Kultur zunächst all das bezeichnet, was der Mensch durch sein Denken und Schaffen hervorbringt, so gehören damit neben Sprache, Religion und Kunst gerade auch Wissenschaft und Technik sowie Medizin zum Kern einer jeden Kultur. Heilkunde beginnt in diesem Sinne in dem Moment, wo der Mensch Kulturwesen wird. Heilkunde und Kultur sind damit untrennbar miteinander verbunden.
}

Obwohl die Medizin als Teil unserer Kultur ohne jeden Zweifel eine der größten kulturellen Errungenschaften darstellt, werden wir im gegenwärtigen Gesundheitssystem mit einer paradoxen Situation konfrontiert: Es hat sich nämlich ein System herausgebildet, das nur noch wenig mit seinen antiken Vorbildern der Heilkunde bzw. Heilkunst zu tun hat und losgelöst ist von Geist und Bewusstsein, durch die es - historisch betrachtet - erst geschaffen wurde. Ein System also, das sich verselbstständigt hat und nun dem Menschen als etwas Äußeres entgegentritt, ihn zunehmend sich seiner selbst entfremden lässt. Heilmittel werden in diesem System isoliert als bloße Techniken und getrennt von ihrem kulturellen Bedeutungsrahmen genutzt; der kranke Mensch wird zunehmend zum Objekt des Systems und seiner Rolle als Protagonist der eigenen Heilung beraubt. Es entsteht eine „sprachlose Medizin“ in der Konzepte wie Bewusstsein, Geist und Kultur keinen Platz mehr haben.

Ivan Illich hat diesen Prozess der Entkulturalisierung der Medizin bereits in den 7oerJahren des letzten Jahrhunderts in einer Kritik ausführlich dargestellt und als „Medikalisierung des Lebens “ bezeichnet. Er hat die These aufgestellt, dass das Gesundheitssystem in westlichen Gesellschaften ein Hemmnis für ein gesundes Leben darstellt und beschreibt eine Iatrogenesis, also eine Schädigung durch die Medizin, auf drei Ebenen:

1. eine klinische Iatrogenesis, die „die organische Fähigkeit zur Lebensbewältigung durch heteronome Verwaltung ersetzt",

2. eine soziale Iatrogenesis, „bei der die Umwelt jener Bedingungen beraubt wird, die es (Menschen) ermöglichen, ihre eigene innere Verfassung und ihr Milieu selbst zu beherrschen" sowie

3. eine kulturelle Iatrogenesis, die einsetzt, „sobald der Medizin-Betrieb den Willen der Menschen schwächt, ihre Realität zu erleiden." (Illich 1995, S. 91)

Nach Illich (1995, S. 92) entwickelt ursprünglich jede Kultur „ein Gefüge von Einstellungen zu Schmerz, Krankheit, Schwäche und Tod“ und erfüllt eine Art hygienische Funktion der Art, dass Mittel und Praktiken entwickelt werden „um Schmerz erträglich, Krankheit oder Schwäche verstehbar und den Schatten des Todes sinnvoll zu machen“ (Illich 1995, S: 93). Traditionelle Kulturen haben es seit jeher verstanden ihren Mitgliedern verbindliche Programme der Gesundheitspflege anzubieten: 
„,ür Essen, Trinken, Arbeiten, Atmen, Lieben, Politikmachen, sportliche Betätigung, Singen, Träumen, Kämpfen und Leiden“ (Illich 1995, S: 93).5

In solchen traditionellen Kulturen zeigt sich noch deutlich, dass Natur und Kultur ineinander übergehen und eine Abgrenzung zwischen beiden keineswegs einfach ist. So können wir etwa in den Säulen der klassischen Naturheilkunde den Faktor Kultur deutlich erkennen, wenn z.B. von Körper- oder Leibeskultur sowie Ernährungskultur die Rede ist. Wir meinen dann wohl am ehesten gesunde und naturgerechte Ernährung bzw. Bewegung, die die Selbstheilungskräfte wirken lässt, d.h. wir trennen gerade nicht.

Illich (1995, S. 93) zeigt nun, dass der moderne Medizinbetrieb die hygienischen Funktion traditioneller Kulturen untergräbt und die Entstehung neuer Kulturprogramme verhindert, „die Verhaltensmuster für Selbstbehandlung und Leiden bieten könnten." Konsequenterweise verwendet er sodann den Begriff Kultur nur noch für traditionelle Kulturen, die über Kulturprogramme für die Gesundherhaltung bzw. die Bewältigung von Krankheit verfügen - den modernen Medizinbetrieb hingegen bezeichnet er als „kosmopolitische Zivilisation“ bzw. „Medizintechnokratie“.

Mit den kurzen Ausführungen ist vielleicht deutlich geworden, dass es möglich ist, den Begriff Kultur sinnvoll in ein Konzept von Heilkunde einzubinden. Noch ist es aber zu früh, von einer eigenständigen Kulturheilkunde zu sprechen. Zwar stimmen die meisten Akteure im Gesundheitssystem heute darin überein, dass Gesundheit und Krankheit nicht nur biologische, sondern immer auch psychische, soziale und geistige Aspekte umfassen - damit gleichzeitig Natur und Kultur tangiert werden. Aber im Alltag von Forschung und Klinik überwiegen immer noch eindimensionale Betrachtungen aus biomedizinischer bzw. psychologischer oder soziologischer Perspektive, die Bewusstsein und kulturelle Faktoren gar nicht oder zu wenig berücksichtigen.

Es kann daher sicher nicht schaden, Gesundheit und Krankheit, Medizin und Heilung (besonders Selbstheilung) auch kulturwissenschaftlich weiter zu denken. Begriffe wie Mensch, Körper und Leib, Bewusstsein und Psyche sowie Geist und Seele gehörten bislang ohnehin zur Domäne der Kulturwissenschaften, wenngleich sie den Kern der Medizin betreffen, die wissenschaftshistorisch betrachtet immer auch eine anthropologische Disziplin war. Eine Erweiterung des eher naturwissenschaftlichen Blickwinkels der Medizin durch die Kulturwissenschaften entspricht schließlich auch der Stellung der Medizin als praktische und polyparadigmatische Wissenschaft, die sich vom Handlungsziel her (,Kranken zu helfen') definiert ${ }^{6}$. Und diese, d.h. die Kranken, wünschen sich zunehmend eine auf ihre Bedürfnisse zugeschnittene Medizin, die nicht Krankheiten sondern den einzelnen Menschen in den Mittelpunkt rückt. Gerhard Danzer (2012) hat dazu jüngst in seiner sehr umfassenden Monographie aufgezeigt, dass eine personale Medizin weit über das biopsychosoziale Konzept und die Psychosomatik hinausgeht und im Hinblick auf jeden einzelnen kranken Menschen auch geistig-kulturelle Facetten einbezieht. Hier scheint sich ein Anknüpfungspunkt für eine Kulturheilkunde zu bieten.

Ein kulturwissenschaftlicher Blickwinkel auf die Medizin ermöglicht auch in forschungsmethodischer Hinsicht eine bessere Berücksichtigung der Anforderungen

5 Illich führt weiter aus: „Heilen ist meist eine traditionelle Art und Weise, Menschen zu pflegen und zu trösten, während sie genesen; und Krankenpflege ist meiste ein Form der Toleranz, die den Gebrechlichen zuteil wird." (1995, S. 93)

6 Siehe dazu Wiesing (2004). 
an eine Medizin, die möglichst alle Facetten eines kranken Menschen erfassen möchte. Kulturwissenschaften sind nämlich - anders als die nomothetischen, d.h. auf Gesetzmäßigkeiten ausgerichteten, Naturwissenschaften - idiographisch und „auf Besonderheiten und Individuelles, Einmaliges“ ausgerichtet; sie befassen sich von ihrem Selbsstverständnis her insbesondere „mit immaterieller, sprachlich verfasster, auf Verständnis und Orientierung zielender Auseinandersetzung mit der Welt, mit ,Sinn-Produktion“ und nichtmaterieller Lebensbewältigung“ (Beiner 2009, S. 12-13). Eine ideographische Betrachtung von Heilung ergänzt die ohne Zweifel wichtige nomothetische Perspektive der Medizin auf Krankheit und Wirkmittel. Beide Herangehensweisen sind wichtig und müssen miteinander verbunden werden, um ein vollständiges Bild von dem komplexen Phänomen Heilung zu bekommen.

\section{Was ist überhaupt Kultur?}

\section{„Denn alle Kultur ist Erweiterung des Bewusstseins.“ (C.G. Jung)}

Der Begriff Kultur ist nicht unproblematisch, eine Verwendung ist dennoch weit verbreitet und seine Semantik außerordentlich vielfältig und vage. Dies zeigen bereits einige Wortbildungen mit Kultur im Deutschen: Wir vermissen z.B. bei einem anderen Menschen Kultur, finden etwas kulturvoll bzw. kultiviert oder kulturlos bzw. unkultiviert, unterscheiden gar zwischen Natur- und Kulturvölkern, sprechen von Kulturpflanzen, von Kultivierung und Rekultivierung, wir kennen eine Kulturindustrie und eine Populärkultur, aber auch eine Hochkultur (mit Kunst, Religion und Wissenschaft als Kern), wissen was ein Kulturprogramm, eine Kulturnationen und ein Kulturmensch ist, versuchen die Kultur eines anderen Landes oder einer anderen Epoche zu verstehen und verwenden Bezeichnungen wie Körperkultur, Stammeskultur und Unternehmenskultur nebeneinander.

Die Kette ließe sich fortsetzen und macht deutlich, dass im Sprachgebrauch das Wort Kultur für verschiedene Zwecke funktionalisiert wird. Es wird sowohl normativ als auch deskriptiv genutzt, kann Positives und Negatives bezeichnen, zeit- und raumbezogen sein7, es ist Prozess und Ergebnis zugleich, dient als Wertbegriff (z.B. zur Identitätsbildung) oder auch zur Abgrenzung bzw. als Gegenbegriff (z.B. die eigene Kultur gegenüber der Barbarei der Anderen). Selbst in den Kulturwissenschaften gibt es keinen einheitlichen Kulturbegriff, sondern vielmehr eine Vielfalt von Forschungsrichtungen und hunderte von Definitionsversuchen.

Übereinstimmung gibt es wohl lediglich in der Auffassung, dass der Begriff Kultur „nicht zu definieren ist“ bzw. „sich jeder Definition entzieht“ - wie es bei Baecker 2000, S. 33) heißt: „Wer es trotzdem versucht, zeigt damit nur, dass er dem Begriff nicht gewachsen ist." Es soll daher hier auch kein weiterer Definitionsversuch gewagt werden, vielmehr soll über die Etymologie und Begriffsgeschichte ein Einblick gegeben werden, wie facettenreich dieser Begriff ist und wo Anknüpfungspunkte zur Thematik Kulturheilkunde festgemacht werden können.

Die spachliche Herkunft des deutschen Wortes Kultur verweist - so viel ist bekannt und anerkannt - auf das Lateinische: colere und davon abgeleitet cultura, Das Verb colere bedeutet ,pflegen', ,den Acker bestellen“ aber auch ,verehren“ und ,anbeten‘.

7 Die Kultur einer Epoche, die Kultur eines Landes, einer Nation, einer Gesellschaft. 
Die Verbindung zur Natur ist zunächst agri cultura durch die Bezogenheit auf den Ackerbau noch deutlich sichtbar. Aber schon ältere Bestimmungen (etwa bei Cato) verweisen neben Bodenkunde und Ackerbau auch auf Bedeutungsnuancen wie Lebensstil, Politik, Sozialkunde, Ernährung und Medizin bzw. verbinden diese miteinander. Schließlich wird bei Cicero die cultura animi erwähnt, d.h. die Pflege des Geistes.

Der Begriff Kultur bezieht sich wohl schon früh auf etwas sowohl Äußeres als auch Inneres zugleich. Beides lässt den Menschen aus dem Tierreich hervortreten. So bestellt der Mensch die Natur mit selbst hergestellten Werkzeugen und sorgt damit über das Heute hinaus für die Zukunft. Dadurch schafft er überhaupt erst die Voraussetzungen für jede Weiterentwicklung von Technik, Wissenschaft und Kunst, Spiel und Unterhaltung. Und er pflegt eben auch den Geist, sucht nach Sinn und Bedeutung, beschäftigt sich mit dem Transzendenten und verehrt das Göttliche - kultiviert sich damit selbst.

Ausgehend von dieser etymologischen Herleitung umfasst Kultur all das, was Menschen durch ihre Tätigkeit und durch ihr Denken hervorbringen und gezielt weitergeben sowie vorausschauend und reflektiert bewahren, indem sie die Natur (das Äußere) sowie sich selbst (das Innere) für bestimmte Zwecke bearbeiten. Die Natur bearbeiten Menschen mit Werkzeugen, die sie selbst produzieren. Sich selbst bearbeiten sie durch die Produktion von Wissen, Kompetenz und Sinn sowie die Beschäftigung mit dem Numinosen und Transzendenten. ${ }^{8}$

Beide Komponenten des Begriffes Kultur finden später vor allem in der Philosophie von Immanuel Kant ihren Ausdruck, der

„die Befreiung des Menschen vom Naturzwang durch die Beherrschung der äußeren Natur mittels Technik und Wissenschaft sowie die Bearbeitung der inneren Triebnatur durch Disziplin und Selbstkontrolle“ forderte (Treichel 2012, S. 19).

Bei Kant findet sich entsprechend ein ganzes Programm der Selbstkultivierung des Menschen, was auch später in der Entwicklung der Naturheilkunde aufgegriffen wurde. ${ }^{9}$

Bezogen auf Cesundheit und Krankheit kann die kurze Diskussion des Kulturbegriffs vielleicht helfen zu verstehen, dass Heilung im Sinne einer Kulturheilkunde nicht nur im Äußeren ansetzt durch eine Bearbeitung von Umgebung und Umwelt sowie das Erschaffen von Artefakten als Heilmittel, sondern immer auch im Inneren anknüpft und auf eine Selbstkultivierung des Menschen setzt: Der wichtigste Protagonist der Heilung ist nämlich der kranke Mensch selbst - eine Sichtweise die im Folgenden unter Bezug auf die Thematik Schmerzen vor allem aus der Sicht der Philosophie Hans-Georg Gadamers diskutiert werden soll.

8 Hier sei weiter nicht diskutiert, wo und wann die menschliche Kultur beginnt: schon mit dem Ackerbau und dem Herstellen von Werkzeugen, dem Bestatten von Toten und Heilen von Kranken oder erst mit Religion und dem Suchen nach Sinn, mit Kunst und Musik oder der Fähigkeit zu Muße und Spiel, mit Moral, Werten, Normen und Gewissen oder mit der Fähigkeit zur Selbstreflexion sowie mit Sprache und Literatur? Alles ist wichtig und miteinander verknüpft.

9 Schröder/Mildenberger (2013): Das Kantsche Programm der Selbstkultivierung findet sich vor allem in seinen Schriften „Über Pädagogik“ (1803) und „Anthropologie in pragmatischer Hinsicht“ (1798), bestimmt jedoch auch die Struktur seines Hauptwerkes, wie es in den drei „Kritiken“ vorliegt. Selbstkultivierung (sozusagen Kulturheilkunde aus Sicht des Patienten) geschieht nach Kant durch Disziplinierung, Zivilisierung und Moralisierung, die er nicht nur als drei Weisen der Selbstkultivierung, sondern auch als drei Phasen oder Stufen der Bildung des Menschen zum Menschen ansieht." 


\section{Der Schmerz als Bewusstseins- und Kulturphänomen}

„Verstehe, oh du Wahrheitssuchender, dieses Prinzip: Nur der, der Schmerz hat, kann verstehen." (D. Rumi, Persische Dichtkunst)

Das Leben beginnt im Schmerz und Schmerzen begleiten uns durch das ganze Leben hindurch bis zum Sterben. ${ }^{10}$ Erst der Tod scheint dem Schmerz Einhalt zu gebieten. Das Schmerzempfinden aber ist für menschliche Leben konstitutiv. Es lässt uns freilich auf eine nicht erwünschte Art - spüren, dass wir leben und stellt uns gleichzeitig eine Aufgabe. Grundsätzlich ist der Schmerz zwar keine Krankheit, sondern allenfalls Symptom einer Krankheit. Er gehört wie Puls, Blutdruck, Temperatur und Atem sogar zu den Vitalzeichen des Menschen ${ }^{11}$, lässt uns unserer Körperlichkeit bewusst werden und trägt „über die unmittelbar negative Valenz des Erlebens hinaus eine äußerst wichtige Funktion (...): nämlich den Erhalt bzw. die Wiederherstellung der körperlichen Unversehrtheit des Organismus zu gewährleisten“ (Kröner-Herwig 2007, S. 10). ${ }^{12}$

Schmerz stellt als körperliches Phänomen durchaus etwas Objektives, jedoch nicht etwas wirklich messbares, dar; zugleich hat er etwas zutiefst Subjektives und betrifft psychische und seelische Aspekte, ist daher auch nur ganzheitlich zu begreifen und $z$ bewältigen. Gerade der chronische Schmerz zeigt wie tief und komplex Krankheit und Gesundheit mit Sprache sowie Kultur verwoben ist. Als Empfindung ist Schmerz auch eine sprachlich-kulturelle Konstruktion ${ }^{13}$, wobei ich hier auf die besondere Rolle der Sprache nicht weiter eingehen werde. ${ }^{14}$ Des Weiteren ist Schmerz unmittelbar auch ein Phänomen des Bewusstseins ${ }^{15}$.

Im Deutschen hat das Wort Schmerz zwei Grundbedeutungen, die sowohl die körperliche als auch die seelische Dimension zum Ausdruck bringen. Wir unterscheiden:

1. eine „infolge Krankheit, Verletzung, Einwirkung schädlicher Reize wahrgenommene sehr unangenehme, peinliche körperliche Empfindung“ als schmerzhaft und

2. eine „durch etwas, was jemandem widerfahren ist, verursachte sehr unangenehme psychische Empfindung “ als schmerzlich. (Handwörterbuch der deutschen Gegenwartssprache, 1984).

Aber auch der somatische Schmerz lässt sich nicht auf etwas rein Physiologisches oder nur auf einen Reiz bzw. eine bloße Nozizeption begrenzen. Denn als Phänomen des Bewusstseins ist die Reaktion bzw. sind Einschätzung, Wahrnehmung, Sinnge-

10 Allerdings muss Sterben nicht unbedingt schmerzhaft und auch nicht schmerzlich sein.

11 Die Ethik-Charta der DGSS sieht den Schmerz als „fünftes Vitalzeichen“ und fordert wie bei Puls, Blutdruck, Temperatur und Atem ein regelmäßiges Messen zur Frühdiagnostik.

12 Erst der „chronische Schmerz verliert die Warnfunktion völlig und wird damit vom Symptom zur Krankheit“" (Kröner-Herwig 2007).

13 Siehe dazu vor allem Lalouschek (2010) und Müller-Busch (2007).

14 Gleichwohl lassen sich dazu interessante Fragen stellen: Existiert Schmerz überhaupt außerhalb der Sprache? Wie beeinflussen die sprachlichen Ausdrucksmöglichkeiten (Benennung bzw. Bezeichnung und dazugehörende Begriffsinhalte) unser Schmerzempfinden und unsere Bewältigungsstrategien?

15 Diese Position wird auch in der Ethik-Charta der DGSS vertreten: „Schmerz ist ein Bewusstseinsphänomen, das sich in den Dimensionen der Wahrnehmung, des Verhaltens und Erfahrung ausdrückt.“ (S. 5) Die Definition von Schmerz lautet entsprechend: „Schmerz verweist nicht nur als Symptom auf die Erregung schmerzvermittelnder (nozizeptiver) Strukturen. Er ist ein Phänomen, das eine physiologische Dimension sowie eine Bewusstseins- und eine Gefühlskomponente hat, die die Intensität und Art des Schmerzerlebens und des Schmerzverhaltens bestimmen. Hinzu kommt, dass die individuelle Schmerztoleranz auch von kommunikativen Gewohnheiten, historischen und psychosozialen Aspekten beeinflusst wird. “ 
bung sowie Ursachenzuschreibung und Bewältigung unterschiedlich und von kulturellen Faktoren und vom Kontext abhängig. ${ }^{16}$

Kohnen (2003) unterscheidet aus ethnomedizinischer Sicht entsprechend zwischen Schmerzhaftigkeit, womit das eher das Körperliche gemeint ist, und Schmerzlichkeit, womit die emotional-seelische Bewertung hervorgehoben werden soll. In der Schmerztherapie geht es nach Kohnen nicht zuletzt auch darum, dem Schmerz die schmerzliche Komponente zu nehmen.

Figge (1980, S. 28) stellt hinsichtlich des Wortes Schmerz eine semantische Verwirrung fest, da mit dem gleichen Ausdruck Bewusstseinsinhalt, Verhalten und sensorische Aktivität gemeint sein können. Er schlägt daher eine Unterscheidung von Nozizeption und Schmerz vor. Der Begriff Nozizeption wäre dem Vorschlag von Figge gemäß auf direkte „Folgen der Reizung von Nozizeptoren“ zu begrenzen. Mit Schmerz wiederum wäre dann eine individuelle „Qualität von Wahrnehmungserlebnissen und anderen Bewusstseinsinhalten“ gemeint, wobei Bewusstseinsinhalte (...) stets das Produkt eines vorangegangenen unbewussten Verarbeitungs- und Bewertungsprozesses sind:

„Nozizeptionen (werden) unter bestimmten Umständen zu Schmerz, z.B. nämlich dann, wenn sie aufgrund des subjektiven Bewerbungsprozesses mit Angst verknüpft sind.“

Ein solches Verständnis von Schmerz wird durch neuere Beiträge aus der Schmerztherapie bekräftigt. So heißt es bei Traue (2008, S. 4):

„Der Schmerz ist ja niemals nur ein sensorisches Ereignis, sondern ein subjektives Erleben, bei dem sich zahlreiche Elemente und Prozesse zwischen Schmerzquelle und Schmerzempfindung erstrecken, die den Schmerz filtern und seine Intensität verkleinern oder vergrößern, seine Wirkung verlangsamen, dämpfen oder beschleunigen."

Und Wörz (2007, S. 14-15) bestätigt, dass „Merkmale der Nozizeption (...) unzuverlässige Indikatoren für das Auftreten von Schmerz" sind und eine morphologische Schädigung nicht notwendig und auch nicht hinreichend für Schmerzempfindung ist:

„Die angemessene Schmerzdiagnostik muss daher vom Erleben der Betroffenen ausgehen und nicht von der im Somatischen ablaufenden Nozizeption."

Auf dieser Crundlage bildet folgender Definitionsversuch von Schmerz im Lexikon von Stubbe (2005) aus ethnomedizinischer Sicht eine gute Grundlage für die Kulturheilkunde:

„Eine grundlegende unangenehme Empfindung, die dem Leib zugeschrieben wird und dem Leiden entspricht, das durch die psychische Wahrnehmung einer realen, drohenden oder phantasierten Verletzung hervorgerufen wird.S(chmerz) wird als eine spezifische Erfahrung betrachtet, die zwischen Emotion und Sinnesempfindung steht. Im übertragenen Sinn auch eine Gefühlsqualität psychischer Vorgänge, die sich in einzelnen Fällen von konkreten körperlichen S(chmerzen) nicht eindeutig abgrenzen lässt.“

Aus philosophischer Sicht hat nun besonders Hans-Georg Gadamer die Bedeutung von Kultur und Bewusstsein im Umgang mit Schmerzen diskutiert und einen grundlegenden Beitrag zu einer Kulturheilkunde der Schmerzen geleistet, auf den daher ausführlicher eingegangen werden soll. Auf Einladung der Heidelberger Orthopädi-

16 So kann Schmerz mehr oder weniger billigend in Kauf genommen werden, weil er in einem bestimmten Kontext mit Sinn verbunden wird oder mit besonderen Strategien bewältigt werden kann: bei ärztlichen Behandlungen (gerade auch aus ästhetischen Gründen), im Sport (insbesondere in Wettkämpfen), bei Initiationen und anderen Ritualen. Schmerzzufügung kann auch ein Instrument der Sanktionierung bzw. Disziplinierung in der Erziehung (in Familie und Gesellschaft) sowie psychopathologisch sein (etwa um sich selbst fühlen zu können). 
schen Universitätsklinik trug Gadamer im Jahr 2000 vor einem medizinischen Fachpublikum „Einschätzungen eines Philosophen“ zur Thematik „Schmerzen“ vor. In diesem letzten öffentlichen Vortrag stellte sich Gadamer - im Alter von 100 Jahren und trotz starker Schmerzen - der Diskussion mit den Medizinern, die er zum Nachdenken über ihre eigene Praxis brachte. Schiltenwolf (2003, S. 5), der Chef der Klinik, drückte das so aus: Gadamer beschrieb

„den Schmerz als eine lebenslange Aufgabe (...), die, weit entfernt ein Makel oder gar Deformation des leidenden Individuums zu sein, sich vielmehr dem strukturell annähert, wasihn zeitlebens beschäftigt hat: der Hermeneutik.“

Vieles was Gadamer dann in freier Rede hervorbrachte klingt heute sehr modern und erinnert an Konzepte wie Selbstheilung(-skräfte) sowie Selbstwirksamkeit, Krankheit als Zeichen, Ressourcenorientierung, Achtsamkeit und Imagination. Manches ist mit neuesten neurowissenschaftlichen Erkenntnissen vereinbar. ${ }^{17}$

Crundlage der „Einschätzungen“ von Gadamer war übrigens nicht die theoretische Beschäftigung mit dem Forschungsstand der einschlägigen Wissenschaften, vielmehr ging er einerseits von seiner eigenen Betroffenheit ${ }^{18}$ aus und andererseits von der von ihm entwickelten Hermeneutik. Wie in der Hermeneutik, so geht es ihm auch bei dem Thema Schmerz um den „Akt des Verstehens“. Schiltenwolf (2003, S. 15) bringt die Argumentation von Gadamer auf den Punkt:

„Nein, Schmerz sei keine Frage nach der besten Medizin, sondern eine Frage an den Betroffenen selbst. (...) Nein, keine Medizin, sondern die Aufgabe im Schmerz lösen lernen. Der Betroffene ist selbst der Protagonist der Heilung."

Im Verständnis von Gadamer gehören Aushalten und Befreiung von Schmerzen „seit jeher zu den Erfahrungen des menschlichen Lebens“ ohne dass für „schmerzhafte Zustände und deren Linderung (...) im allgemeinen medizinische Hilfe in Anspruch genommen werden muss.“ (2003, S. 22) Vielmehr komme es darauf an, dass „die natürlichen Formen der Behandlung von Schmerzen“ sowie „die althergebrachten Heilverfahren gepflegt werden sollten.“ (2003, S. 23), wobei sich Gadamer auf seinen langjährigen Arzt und Freund Paul Vogler bezieht, der die dritte Professur für natürliche Heilverfahren an der Charité innehatte.

Gadamer ist sich bei seinen Ausführungen der Vielfältigkeit ${ }^{19}$ und der Totalität ${ }^{20}$ des Phänomens Schmerz durchaus bewusst. Gleichwohl ist es erforderlich Schmerz - ganz egal wie groß er ist - zu verwinden, womit er eine „Meisterung der Schmerzen“ meint. Eine Möglichkeit dafür sieht er

17 So wirft Hennigsen (2008, S. 22-24) einen „Blick auf die Neurobiologie von Schmerzen“ und zeigt auf der Grundlage des Phänomens der erfahrungsabhängigen Neuroplastizität und der Möglichkeiten der Bildgebung, dass a) „neurophysiologische Korrelate für psychologische Einflüsse auf Schmerz“ nachgewiesen werden können, b) „psychischer (per Hypnose induzierter) Schmerz (...) ähnliche neurophysiologische Korrelate wie körperlicher Schmerz“ hat. Sein Fazit lautet: „Neurobiologische Befunde helfen also, den engen Zusammenhang von psychischen und körperlichen Aspekten bei der Wahrnehmung und Verarbeitung von Schmerz zu verstehen. Wir haben uns heute vom dualistischen Verständnis des Schmerzes entfernt: Schmerz ist immer psychosomatisch." (2003, S. 28)

18 Schmerzen begleiteten Gadamer von der Jugend an bis ins hohe Alter. Mit 22 Jahren litt er an Polio und betrieb im Bett seine philosophischen Studien. In den Ruhestunden las er das Gesamtwerk von Jean Paul („so an die 20 Bände“): „Schließlich gingen die Schmerzen zurück und es blieb nur eine Schwäche meiner Beine, die mich jahrelang hat Sport treiben lassen. (...) Erst in meinem 75. Lebensjahr musste ich das Tennisspielen aufgeben und in all meinen Heidelberger Jahren waren mir die schönen Waldwege im Odenwald sämtlich gut vertraut.“ (...) „)etzt freilich muss ich an Stöcken gehen und auf einem Standrad trainieren, um überhaupt noch auf meinen eigenen Beinen gehen zu können.“ (2003, S. 24-25)

19 Gadamer nennt den ersten „Schmerzensschrei des gerade Geborenen“ und die „Gebrechen, den ,leisen', doch konstanten Schmerz der Altersbeschwerden“ (2003, S. 26-27).

20 So heißt es u.a.: „denn wir sind im Schmerz und können ihn nicht von uns trennen. Der Schmerz umgreift gleichsam unser Leben und fordert uns beständig neu heraus." (2003, S. 27). 
„durch das eigene Sich-Wehren gegen den Schmerz in diesen einzugreifen, indem man sich dem ganz hingibt, was einen ganz erfüllt. Nichts lässt den Schmerz am ehesten erträglich werden als das Gefühl, es geht mir etwas auf, mir fällt etwas ein. (...) In diesem Sinne ist der Schmerz (...) vielleicht die größte Chance, endlich mit dem, fertig zu werden', was uns aufgegeben ist." (2003, S. 27)

Gadamer sieht - ähnlich wie Illich - eine große Gefahr, dass durch übermäßige und vorschnelle Interventionen des Arztes gerade diese Chance vertan wird, „dass diese Kräfte unterschätzt werden und damit auch - verständlicherweise - unsere Fähigkeiten nicht mehr zur vollen Entwicklung gelangen“. Die „Freude des Gelingens, des Wachseins und des Hingegebenseins“ - für ihn „das beste Medikament (...), das uns die Natur an die Hand zu geben weiß“ - lässt uns vielleicht am besten und immer wieder diesen schönen „Zustand der Selbstvergessenheit“ erreichen, womit Gadamer die „Verborgenheit der Gesundheit“ meint (2003, S. 27-28).

An anderer Stelle geht Gadamer (1993, S. 102) - unter Bezug auf Heraklit - noch deutlicher auf das „Problem des Wegnehmens und Wegmachens“ ein:

„Man beachte, wie Schmerz etwas anderes wird und wie Leiden, das einem durch Schmerz auferlegt ist, etwas anderes wird, wenn es nicht längervon der Erwartung des Aufhörensoder von der Gewissheit, dass es einem weggenommen werden kann, begleitet ist. (...) Man nimmt etwas dagegen und dann ist es weg."

Da der Schmerz aber auch einen tieferen Sinn für einen Leidenden haben kann, kann ein unreflektiertes Wegnehmen und Wegmachen dazu führen, dass ein Problem ungelöst bleibt. ${ }^{21}$ Gadamer weist dem Arzt daher eine maieutische Funktion zu, d.h. er soll seine Rolle darin begrenzen, den Kranken im Bewusstwerden seiner Kräfte der Selbstheilung zu unterstützen, die sich eben auch über den Schmerz vollzieht. ${ }^{22}$ Dabei geht es ihm vor allem darum „kraft dieser Belebung das eigene Können und das eigene Gelingen wieder erfahrbar zu machen.“ (2003, S. 29)

Schiltenwolf fasst die Bedeutung Gadamers Ausführungen so zusammen, dass der Patient als Subjekt erreicht werden muss und eine „Schmerzbehandlung über den Patienten hinweg, quasi als mechanische Handlung am defekten Objekt wenig Sinn macht“ (2003, S. 14). Er schließt nicht aus, dass die Lösung der Aufgabe, die der Schmerz dem Patienten nach Gadamer stellt, letztendlich auch „in die Loslösung und Überwindung der Therapiebedürftigkeit münden“ kann (2003, S. 14).

Was für einen Beitrag könnte Kulturheilkunde zur Behandlung von Schmerzpatienten leisten? Wie könnte sie Patienten bei der Bewältigung ihrer Schmerzen unterstützen? Der Forschungsstand der modernen Schmerztherapie zeigt, dass keine der bisherigen Behandlungsmethoden „allein wirksam genug ist, um mit dem Phänomen Schmerz ausreichend umzugehen."(Nöcker-Ribaupierre 2008, S. 7). Da Schmer-

21 Aus psychotherapeutischer Sicht wird dies im Nachwort von Hermann Lang (2003, S. 45) bestätigt: „Würde man im Falle einer psychosomatischen Schmerzerkrankung sofort und radikal medikamentös intervenieren, hätte der Patient wohl keine Chance mehr, die psychischen bzw. psychosozialen Probleme, die hier vielleicht ins Organische verschoben wurden, zu bearbeiten und zu lösen. Das war ja die große Entdeckung der modernen Psychotherapie, herausgefunden zu haben, dass seelische oder körperliche Symptome einen ,Sinn` haben können, sie im Daseinsgang des betroffenen Menschen als ein sinnvolles Geschehen zu verstehen sind, sie in bestimmten psychosozialen Belastungen, Konflikten gründen können. “

22 „Das müssen wir Menschen lernen, dass alle gesundheitlichen Störungen, Wehwehchen und selbst alle Infektionen in Wahrheit Winke sind, das Angemessene, die Balance des Gleichgewichts, wiederzugewinnen. Am Ende gehört beides zusammen, Störung und ihre Überwindung. Das macht das Wesen des Lebens aus. Von hier aus erhält der Begriff der Behandlung seine kritische innere Begrenzung. Der behandeInde Arzt weiß das recht gut. Er muss sich immer wieder zurücknehmen, um mit vorsichtiger Hand den Patienten zu leiten und seine Natur wieder zu sich selbst zurückkehren zu lassen.. (Gadamer 1993, S. 171) 
zen als Resultat neuroplastischer, psychischer und sozialer Prozesse ein multidimensionales Phänomen darstellen sowie Leiden und Schmerzerleben immer auch kulturell geprägte Bewusstseins- und Kulturphänomene sind, muss eine umfassende Therapie einerseits multidimensional und andererseits individuell angelegt werden. Gefordert ist eine interdisziplinäre Zusammenarbeit von Medizin, Psychologie und Kulturwissenschaften unter Einbeziehung von Musik, Kunst und Etnomedizin. Und hier liegen auch Gadamers (1993, S. 104-105) Hoffnungen: „dass es uns gelingen möchte, aus dem Erbe aller Menschheitskulturen, wie es uns langsam aus planetarischen Weiten und Fernen erreicht, so viel zu lernen, dass wir Bewältigung unserer Abhängigkeiten und Überwindung unserer Störungen durch Bewusstmachung erreichen!“

\section{Heilung als natürlicher und kultureller Prozess}

Die Natur heilt - Kultur und Heilkunst geben Anstöße und bilden den Rahmen für heil und ganz sein. (Therapeium - Die Kunst des Heilens)

Ich komme noch einmal auf Ivan Illich zurück für den Kulturen („Systeme von Sinnbedeutungen“) für Zwecke der Heilung von allergrößter Bedeutung sind. Das gegenwärtige westliche Gesundheitssystem „kolonialisiert“ aber traditionelle Kulturen und ihre Programme der Gesundheitspflege zu Gunsten eines Systems von bloßen Techniken. Im Hinblick auf Schmerz argumentiert Illich: „Kultur macht den Schmerz erträglich, indem sie ihn in ein sinnvolles Umfeld integriert; die kosmopolitische Zivilisation löst den Schmerz aus jedem subjektiven oder objektiven Kontext, um ihn zu beseitigen. Kultur macht den Schmerz erträglich, indem sie ihn als Notwendigkeit interpretiert; nur als heilbar aufgefasster Schmerz ist unerträglich. “ (Illich 1995, S. 94)

Behandlung und Bewältigung von Schmerzen erfordern Natur und Kultur in gleicher Weise. Als Naturwesen verfügt der Mensch über alles, was er zur Selbstheilung benötigt; als Kulturwesen kann er mit Hilfe seines Bewusstseins den Prozess der Heilung - wie beim Bestellen des Ackers - durch sein gezieltes Tun im Äußeren und Denken im Inneren anstoßen und begleiten. Heilung wird nicht gemacht - sie geschieht: in einem Feld der Passung und Resonanz zwischen dem kranken Menschen, dem Therapeuten und den gewählten Wirkmitteln sowie auf dem Boden einer unterstützenden Umgebung (Kultur) und Umwelt (Natur). Heilung ist das Zulassen der inneren Kraft der Selbstheilung, wobei der „innere Arzt“ bzw. die Natur heilt - die Heilkunst gibt lediglich Anstöße und stellt einen förderlichen Rahmen. Therapeuten sind in diesem Prozess Begleiter und Geburtshelfer. Natur- und Kulturheilkunde wachsen auf diese Weise wieder zusammen, denn sie gehören zusammen.

Es kann also nicht schaden eine Weile über Kulturheilkunde nachzudenken. Dabei sollte es keine Rolle spielen, ob die bevorzugten spezifischen Wirkmittel eher aus der konventionellen Medizin oder mehr aus der komplementären Medizin stammen. Kulturheilkunde setzt ja gerade nicht in der Modifikation der spezifischen Methode, sondern in der Modifikation der Heilkultur an. Sie wird so - im Verein mit den Bewusstseinswissenschaften - den Graben zwischen den Blöcken in der gegenwärtigen Medizin im Interesse des kranken Menschen überwinden helfen: vielleicht durch eine Kultur des Bewusstseins? 


\section{Literatur}

Baecker D (2000) Wozu Kultur? Berlin: Kulturverlag Kadmos.

Deutsche Gesellschaft zum Studium des Schmerzes e.V. (2007) Ethik-Charta der DGSS. Boppard: Geschäftsstelle der DGSS.

Beiner M (2009) Humanities. Was Geisteswissenschaft macht. Und was sie ausmacht. Berlin: Berlin University Press

Danzer G (2012) Personale Medizin. Bern: Verlag Hans Huber

Figge HH (1989) Schmerz - Urerfahrung oder kulturspezifisches Konstrukt? In: Schmerz - interdisziplinäre Perspektiven. Beiträge zur 9. Internationalen Fachkonferenz Ethnomedizin in Heidelberg vom 6.5.-8.5.1988 Herausgegeben im Auftrag der Arbeitsgemeinschaft Ethnomedizin von Katrin Greifeld, Norbert Kohnen, Ekkehard Schröder. Braunschweig/Wiesbaden: Verlag Friedr. Vieweg \& Sohn. S. 23-32

Gadamer H-G (1993) Über die Verborgenheit der Gesundheit. Aufsätze und Vorträge. Frankfurt am Main: Suhrkamp Verlag

Gadamer H-G (2003) Schmerz. Einschätzungen eines Philosophen. In: Schmerz. Einschätzungen aus medizinischer, philosophischer und therapeutischer Sicht. Heidelberg: Universitätsverlag Winter. S. 21-40

Handwörterbuch der deutschen Gegenwartssprache. In zwei Bänden (1984). Berlin: Akademie-Verlag

Hennigsen P (2008) Ein klinischer Blick auf die Neurobiologie von Schmerzen. In: Musiktherapie und Schmerz. $\mathrm{Hg}$. von Monika Nöcker-Ribaupierre. Wiesbaden: Reichert Verlag. S. 19-30

Herder JG (1784) Philosophie der Geschichte der Menschheit. In: (1887). Sämtliche Werke Bd. 13. Berlin: Weidmannsche Buchhandlung

Illich I (1995) Die Nemesis der Medizin. Die Kritik der Medikalisierung des Lebens. München: Verlag C.H. Beck

Kohnen N (2003) Von der Schmerzlichkeit des Schmerzerlebens. Wie fremde Kulturen Schmerzen wahrnehmen, erleben und bewältigen. Ratingen: PVV

Kröner-Herwig B (2007) Schmerz - eine Gegenstandsbestimmung. In: Kröner-Herwig B, Klinger R, Fretlöh |, Nilges P (Hrsg.) Schmerzpsychotherapie. Heidelberg: Springer-Verlag. S. 7-19

Lalouschek I (2010) Medizinische und kulturelle Perspektiven von Schmerz. In: Florian Menz et al. (Hrsg.) Sprechen über Schmerzen. Linguistische, kulturelle und semiotische Analysen. Duisburg: Universitätsverlag Rhein-Ruhr. S. 15-69

Lang H (2003) Nachwort zu Hans-Georg Gadamer: Schmerz. Einschätzungen aus medizinischer, philosophischer und therapeutischer Sicht. Heidelberg: Universitätsverlag Winter. S. 43-53

Müller-Busch HC (2007) Kulturgeschichtliche Bedeutung des Schmerzes. In: Schmerzpsychotherapie. Hg. von Birgit Kröner-Herwig et al. Heidelberg: Springer-Verlag. S. 151-167

Nöcker-Ribaupierre M (2008) Musiktherapie und Schmerz - eine Einführung. In: Musiktherapie und Schmerz. Hg. von Monika Nöcker-Ribaupierre. Wiesbaden: Reichert Verlag. S. 12-18

Pick L (2011) Kulturelle Partizipation und Gesundheit - Diskussion des Potenzials und der Wirksamkeit von Kultur als Präventions, Reduktions- oder Heilmittel bei Stress oder stressbedingten Erkrankungen. Unveröffentlichte Masterarbeit. Frankfurt (Oder). Kulturwissenschaftlichen Fakultät der Europa-Universität Viadrina

Schiltenwolf M (2003) Editorische Notiz und Vorwort zu Hans-Georg Gadamer: Schmerz. Einschätzungen aus medizinischer, philosophischer und therapeutischer Sicht. Heidelberg: Universitätsverlag Winter. S. 5-17

Schröder H u. Mildenberger F (2013) Kulturheilkunde. Was könnte sie sein und leisten? In: Kultur-Medizin-Gesellschaft. Schriftenreihe des Instituts für transkulturelle Gesundheitswissenschaften. Essen: KVC Verlag. (Im Druck)

Stubbe H (2005) Lexikon der Ethnopsychologie und Transkulturellen Psychologie. Frankfurt am Main IKO-Verlag für Interkulturelle Kommunikation.

Traue HC (2008) Gedanken zur Schmerzpsychotherapie. Eröffnungsvortrag anlässlich der Jahrestagung der DGPSF in Nottwil/Schweiz vom 29. - 31 Mai 2008. Online abgerufen am 09.09.2013: http://www.dgpsf.de/filead$\mathrm{min} /$ user_upload/downloads/Gedanken_zur_Schmerzpsychotherapie_Nottwil_HCT_16-10-08.pdf

Treichel D (2011) Entwicklung des Kulturbegriffs in großen Sprüngen. In: Dietmar Treichel und Claude-Helene Mayer (Hrsg.) Lehrbuch Kultur. Münster: Waxmann Verlag. S. 17-23

Wiesing U (2004) Wer heilt, hat Recht? Über Pragmatik und Pluralität in der Medizin. Stuttgart: Schattauer Verlag.

Wörz R (2007) Schmerz ist ein Bewusstseinsphänomen. In: Schmerztherapie. Nr. 4/2007. S. 14-15 


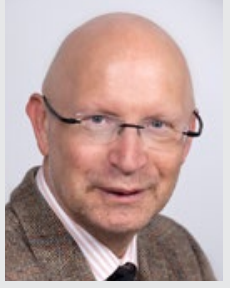

Prof. Dr. Hartmut Schröder

ist Professor für Sprachgebrauch und Therapeutische Kommunikation an der Kulturwissenschaftlichen Fakultät der Europa-Universität Viadrina in Frankfurt (Oder). Er promovierte 1985 an der Universität Bielefeld, erhielt 1990 einen Ruf an die Universität Vaasa in Finnland, wo er den Lehrstuhl für Fachsprachen und Translation leitete. Seit 2010 ist er Direktor des Steinbeis-Transfer-Instituts für Therapeutische Kommunikation und integrierte Therapie der Steinbeis-Hochschule Berlin, wo er in mehreren Studiengängen unterrichtet. 